\title{
Dectin-1-induced RIPK1 and RIPK3 activation protects host against Candida albicans infection
}

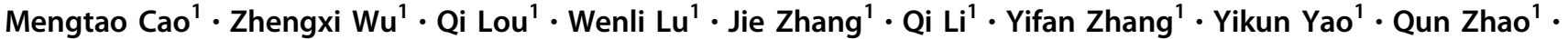 \\ Ming $\mathrm{Li}^{1} \cdot$ Haibing Zhang $\mathbb{D}^{1} \cdot$ Youcun Qian $\mathbb{D}^{1,2}$
}

Received: 5 November 2018 / Revised: 5 March 2019 / Accepted: 21 March 2019 / Published online: 3 April 2019

(c) ADMC Associazione Differenziamento e Morte Cellulare 2019

\begin{abstract}
Necroptosis is a recently defined type of programmed cell death with the specific signaling cascade of receptor-interacting protein 1 (RIPK1) and RIPK3 complex to activate the executor MLKL. However, the pathophysiological roles of necroptosis are largely unexplored. Here, we report that fungus triggers myeloid cell necroptosis and this type of cell death contributes to host defense against the pathogen infection. Candida albicans as well as its sensor Dectin-1 activation strongly induced necroptosis in myeloid cells through the RIPK1-RIPK3-MLKL cascade. CARD9, a key adaptor in Dectin-1 signaling, was identified to bridge the RIPK1 and RIPK3 complex-mediated necroptosis pathway. RIPK1 and RIPK3 also potentiated Dectin-1-induced MLKL-independent inflammatory response. Both the MLKL-dependent and MLKL-independent pathways were required for host defense against $C$. albicans infection. Thus, our study demonstrates a new type of host defense system against fungal infection.
\end{abstract}

\section{Introduction}

Fungal infections severely influence human life quality and are common worldwide health problem. One and a half million people die from invasive fungal infection every year [1]. Especially for individuals with AIDS, fungal infection can develop into disseminated candidiasis and even result in mortality in severe cases [2]. As the limited

These authors contributed equally: Mengtao Cao, Zhengxi Wu, Qi Lou

Edited by H.-U. Simon

Supplementary information The online version of this article (https:// doi.org/10.1038/s41418-019-0323-8) contains supplementary material, which is available to authorized users.

Youcun Qian

ycqian@sibs.ac.cn

1 CAS Key Laboratory of Tissue Microenvironment and Tumor, CAS Center for Excellence in Molecular Cell Science, Shanghai Institute of Nutrition and Health, Shanghai Jiao Tong University School of Medicine (SJTUSM) \& Shanghai Institutes for Biological Sciences (SIBS), Chinese Academy of Sciences (CAS), 200031 Shanghai, China

2 School of Life Science and Technology, ShanghaiTech University, 200031 Shanghai, China antifungal drugs used in clinical application and increased drugs resistance, it is of great importance to investigate how host defenses against fungal infection to develop new strategies and targets to treat fungal infection.

C-type lectin receptors are the pathogen recognition receptors to detect fungus, activation of which initiates host immune response against fungal infection [3]. Dectin-1 receptor is the most well-studied C-type lectin receptor, which recognizes $\beta$-glucan of fungal cell wall [4]. Some key kinases and adaptor proteins have been identified to participate in Dectin-1-mediated signaling. SYK, a Dectin-1 downstream signaling kinase, promotes the formation of CARD9-BCL10-MALT1 complex that triggers NF-кBdependent production of inflammatory cytokines [5]. These inflammatory cytokines can further induce Th1 and Th17 cell responses that have been reported to be critical to control Candida albicans infection [6, 7]. Recently, Dectin-1 activation in dendritic cells can induce caspase-8-dependent inflammasome activation which cleaves pro-IL-1 $\beta$ and pro-IL-18 to mature IL- $1 \beta$ and IL-18 to defend C. albicans infection [8]. Thus, Dectin-1induced signaling can initiate both innate and adaptive immune responses to fight against fungal infection.

Necroptosis is a recently identified form of programmed necrotic cell death [9]. TNF is the most investigated trigger of necroptosis, studies of which lead to the fundamental 
understanding of necroptotic signaling process. The two kinases RIPK1 and RIPK3 and the pseudokinase MLKL are three key proteins of TNF-induced necroptosis [9, 10]. RIPK1 and RIPK3 form complex through their receptorinteracting protein kinase (RIP) homotypic interaction motif (RHIM) after necroptosis triggers like TNF [11-13]. Autophosphorylation of RIPK1 promotes the phosphorylation and oligomerization of RIPK3, which leads to RIPK3 activation [13-16]. Activated RIPK3 then phosphorylates MLKL, resulting in MLKL oligomerization, translocation to plasma membranes and disruption of membranes [17-19]. In addition to TNF, some other stimuli including pathogen-associated molecular patterns (PAMPs) from virus or bacteria have been found to induce necroptosis in diverse cell types recently [1]. While both LPS-induced TLR4 signaling and poly I:C-induced TLR3 signaling utilize the signaling adaptor TRIF to activate the type I interferon-producing pathway, TRIF is also required for the two TLRs-induced necroptosis pathway in which TRIF directly associates with RIPK3 via RHIM domain in macrophages [20]. Interferons including interferon $\alpha$, interferon $\beta$ and interferon $\gamma$ have also been reported to induce necroptosis in epithelial cells and macrophages [21]. Both the TLRs-induced TRIF-dependent necroptosis and the interferons-induced necroptosis have been shown to play vital roles in necroptosis-related embryonic lethality and inflammation [22, 23]. Similar to PAMPs, live viruses and bacteria, such as HSV, influenza virus and Staphylococcus aureus, are reported to induce RIPK3 and MLKLdependent necroptosis, activation of which contributes to the pathogenesis and host defense of those infections [24-27]. However, whether fungal infection can induce necroptosis and what is the potential pathophysiological outcome of necroptosis during fungal infection still remain to be determined.

Here, we showed that both $C$. albicans infection and its sensor Dectin-1 activation triggered the necroptotic signaling cascade of RIPK1, RIPK3 and MLKL and cell death in myeloid cells under the condition of apoptosis blockage. We demonstrated that the Dectin-1-induced necroptosis and cell death is independent on autocrine TNF. We further found that Dectin-1 activation resulted in CARD9 association with RIPK1 to induce necroptosis. Through genetic deficiency of RIPK3 and MLKL as well as RIPK1 kinase inhibition, we demonstrated that the induction of the necroptosis pathway is important for the host to protect against $C$. albicans infection in vivo. Furthermore, we revealed that RIPK1 and RIPK3 potentiated Dectin-1induced MLKL-independent inflammation under apoptosis blockage condition and that this MLKL-independent inflammation also contributed to host defense against $C$. albicans infection. Thus, our results demonstrate that fungus can induce necroptosis in macrophages and the induction of necroptosis signaling is critical for host defense against fungal infection.

\section{Results}

\section{Fungus and its PAMPs induce necroptosis in myeloid cells}

Necroptosis can be triggered by TNF, or FasL, under the condition of apoptosis blockage or deficiency [28]. Some bacteria and virus as well as their PAMPs like LPS and poly $\mathrm{I}: \mathrm{C}$ have also been reported to be able to induce necroptosis in the condition of apoptosis blockage [9]. However, it is still unknown whether fungus and its PAMPs can induce necroptosis. To investigate whether fungal PAMPs can trigger necroptosis, we stimulated macrophages cell line J774a.1 with zymosan or curdlan. We found that costimulation of zymosan or curdlan with the pan-caspase inhibitor zVAD induced J774a.1 cell death (Fig. 1a), while the PAMPs alone did not trigger cell death. Because caspase- 8 has been reported to inhibit other triggers-induced necroptosis [29], we sought to determine whether caspase 8 functions similarly in the PAMPs-induced cell death. Indeed, zymosan alone was enough to trigger cell death in caspase-8 knocked-down J774a.1 cells (Fig. S1a and S1b). Consistent with the results from J774a.1 cell line, cotreatment of zymosan plus zVAD or curdlan plus zVAD induced cell death in peritoneal macrophages (PMs) and bone-marrow-derived macrophages (BMDMs) as well as bone-marrow-derived dendritic cells (BMDCs) (Fig. 1b-d). In addition to the mouse myeloid cells, we also observed that the fungal PAMPs triggered cell death in human THP1-derived macrophages in the presence of caspases inhibitor (Fig. 1e). To further confirm the PAMPs-induced cell death, we stained PMs with propidium iodide (PI). FACS analysis indeed showed that PI-positive PMs increased after costimulation of zymosan plus zVAD or curdlan plus zVAD (Fig. 1f, g). Autocrine TNF has been reported to contribute to necroptosis induced by TLR2 signaling. To explore whether potential autocrine TNF is responsible for the fungal PAMPs-induced cell death, we stimulated $T n f^{-/-}$ PMs with zymosan or curdlan together with zVAD. We indeed observed that TLR2 ligand-induced cell death was dependent on autocrine TNF (Fig. 1h and S1c), consistent with the literature report [30]. However, zymosan or curdlan still triggered cell death in $T n f^{-1-}$ macrophages (Fig. $1 \mathrm{~h}$ and S1c). To further determine whether live fungus can trigger necroptotic cell death, we stimulated macrophages with C. albicans plus zVAD. Though low MOI C. albicansinduced LDH release was less in $\mathrm{Tnf}^{-/}$macrophages, high MOI $C$. albicans-induced LDH release had no difference between WT and $\mathrm{Tnf}^{-/}$macrophages (Fig. 1i), suggesting 
a

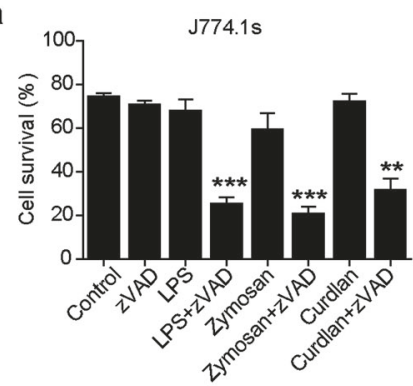

b

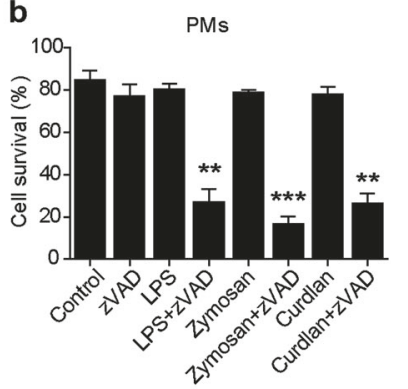

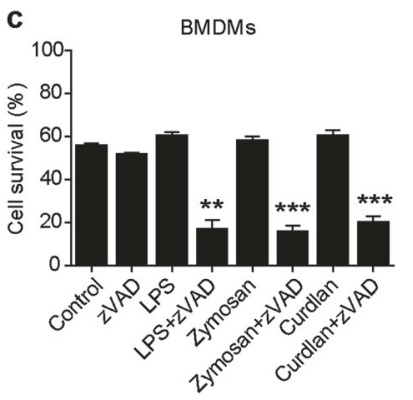
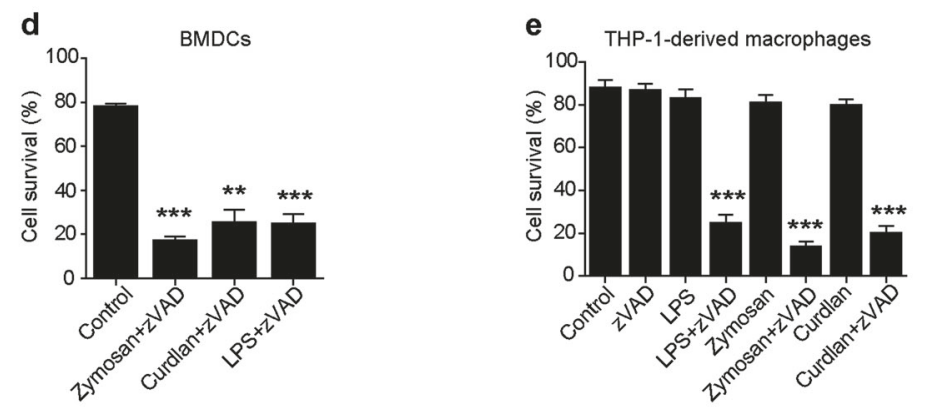

f
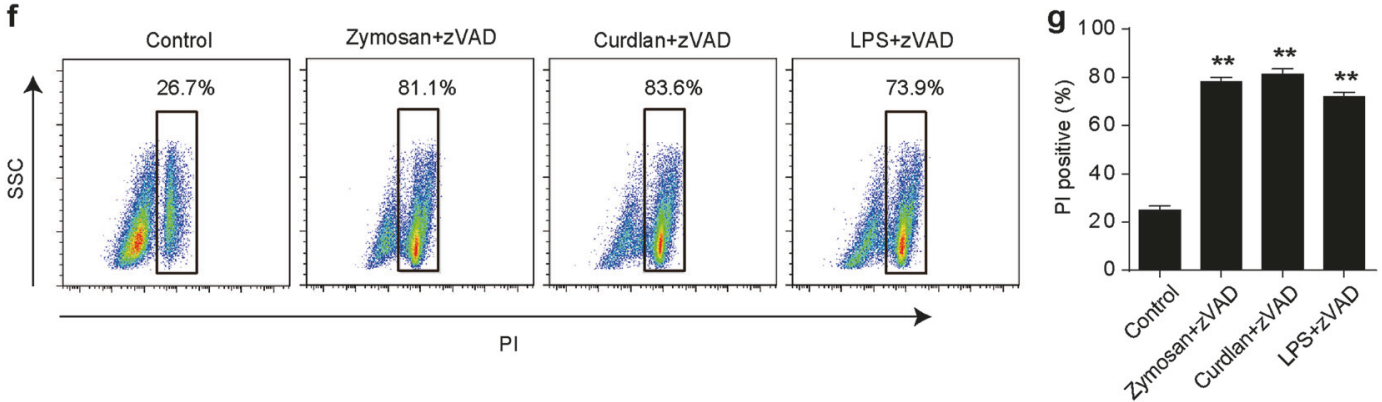

h

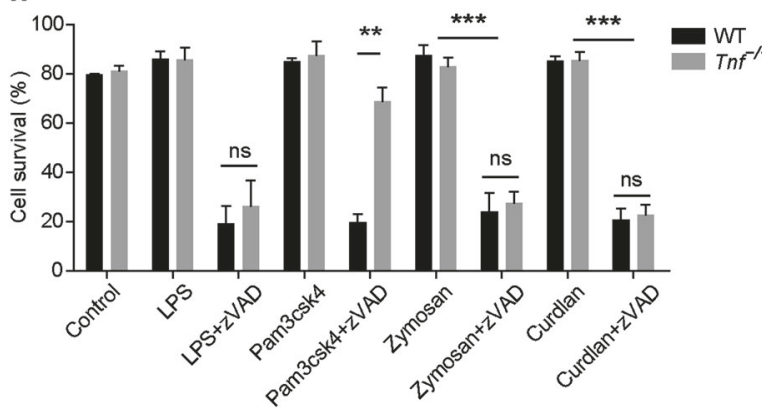

i

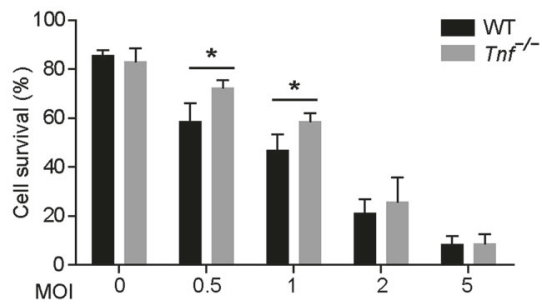

Fig. 1 Fungus and its PAMPs induces myeloid cell death in the presence of caspase- 8 inhibitor. a-e J774A.1 cells (a), peritoneal macrophages (PMs) (b), bone marrow-derived macrophages (BMDMs) (c), bone marrow-derived dendritic cells (BMDCs) (d) and THP-1derived macrophages (e) were treated with the indicated ligands $(25$ $\mathrm{ng} / \mathrm{ml}$ LPS, $100 \mu \mathrm{g} / \mathrm{ml}$ zymosan, $100 \mu \mathrm{g} / \mathrm{ml}$ curdlan) combined with the caspases inhibitor $20 \mu \mathrm{M} z V A D$ or not for $12 \mathrm{~h}$. Cell viability was determined by measuring the released lactate dehydrogenase (LDH). f Peritoneal macrophages (PMs) were stimulated with the indicated ligands and $\mathrm{zVAD}$ for $12 \mathrm{~h}$ and then stained with propidium iodide (PI) and analyzed with flow cytometer (FACS). Representative plots of

C. albicans can induce TNF-independent necroptotic cell death. We further found $C$. albicans and its PAMPs-induced necroptotic cell death did not rely on FasL and TRAIL data are shown. $\mathbf{g}$ The percentage of PI-positive cells are shown from (f). h Peritoneal macrophages from wild-type or $T n f^{-1-}$ mice were treated with the indicated ligands and zVAD for $12 \mathrm{~h}$. Cell viability was determined by measuring released LDH. Data are representative of three independent experiments. i Peritoneal macrophages from wildtype or $\mathrm{Tnf}^{-1-}$ mice were treated with different MOI of C. albicans and zVAD for $12 \mathrm{~h}$. Cell viability as determined by measuring released $\mathrm{LDH}$. Error bars in $(\mathbf{a}, \mathbf{b}, \mathbf{c}, \mathbf{d}, \mathbf{e}, \mathbf{g}, \mathbf{h}$ and $\mathbf{i})$ represent mean $\pm \mathrm{SEM}$. $* \mathrm{p}<0.05 ; * *<0.01 ; * * * p<0.001$; n.s. $p>0.05$ by two-tailed test. See also Figs. S1, S2

(Fig. S2a-S2c), while FasL and TRAIL can trigger necroptosis $[31,32]$. We also found that the fungal PAMPsinduced cell death was independent on MyD88 and Trif 
(Fig. S1d). Collectively, these observations demonstrated that $C$. albicans and its PAMPs could induce myeloid cell death in the absence of caspase- 8 activity.

\section{Fungus and its PAMPs trigger the signaling cascade of RIPK1-RIPK3-MLKL to induce necroptotic cell death}

As the fungus and its PAMPs-induced cell death exhibited necroptotic morphology with swelling and rupture (Fig. S1c), we postulated that RIPK1, RIPK3 and MLKL may participate in the PAMPs-induced cell death. Firstly, we made use of RIPK1 kinase inhibitor Nec-1 and found that Nec-1 blocked the zymosan or curdlan-induced cell death (Fig. 2a). We also found that siRNA-mediated silencing of RIPK3 or MLKL severely reduced the cell death triggered by the zymosan or curdlan in J774.1 cells (Fig. S3a-S3d). In addition, PMs from Ripk $3^{-/-}$mice or $\mathrm{Mlkl}^{-1-}$ mice were protected from the fungal PAMPstriggered cell death (Fig. 2b, c). To further determine whether the signaling cascade of RIPK1-RIPK3-MLKL is required for live fungus-triggered cell death, we stimulated macrophages with $C$. albicans plus zVAD and found that RIPK1, RIPK3 and MLKL were all required for C. albicans-induced cell death (Fig. 2d, f). It has been reported that MLKL can be phosphorylated and translocated to plasma membranes to disrupt their integrity [17, 18]. Similarly, we found that the fungal PAMPs plus zVAD stimulation in PMs induced phosphorylation of MLKL while the ligands alone did not (Fig. 2g). Similarly, we found that the fungal PAMPs triggered the activation of MLKL in human THP-1derived macrophages (Fig. 2h). We further observed that $C$. albicans plus zVAD triggered robust phosphorylation of MLKL in PMs (Fig. 2i). We also found decreased phosphorylation of MLKL in RIPK3-deficient macrophages and Nec-1 pretreated macrophages under the treatment of the fungal PAMPs and zVAD (Fig. 2j, k). Previous studies have implicated $C$. albicans can induce macrophage pyroptosis through Caspase-1 [33, 34]. Because zVAD is pan-Caspase inhibitor, it also can inhibit Caspase-1 function. Consistently, we found that $C$. albicans could induce macrophage cell death and Caspase-1 activation while its PAMPs zymosan and curdlan could not (Fig. S4a and S4b). We further noticed that $C$. albicans with zVAD induced stronger cell death while Caspase-1 was not activated under this condition (Fig. S4a and S4b), suggesting Dectin-1-triggered necroptosis is important for the fungus-induced cell death. In addition, we found that none of RIPK1, RIPK3 and MLKL was required for $C$. albicans-induced Caspase-1 activation (Fig. S4c-S4e). Together, all these data suggest that fungal PAMPs induce RIPK1, RIPK3 and MLKLdependent necroptosis under the condition of apoptosis suppression.

\section{CARD9 mediates the fungal sensor Dectin-1-induced necroptosis activation}

Dectin-1 is the key receptor that senses fungal pathogen infection [35]. The fungal PAMPs like zymosan and curdlan directly activate Dectin-1 signaling to help host defense against fungal infection [36]. We have demonstrated that the fungal PAMPS induced the necroptotic signaling cascade activation of RIPK1, RIPK3 and MLKL. Next we explored if Dectin-1 is the sensor for the fungal PAMPs to induce necroptosis. We found that siRNA-mediated knockdown of Dectin-1 in macrophages made the cells resistant to zymosan or curdlan-induced necroptosis (Fig. 3a, b). We also observed that silence of Dectin-1 in macrophages impaired the fungal PAMPs-induced activation of MLKL (Fig. 3c, d).

Next we tried to explore how the already known Dectin1-triggered signaling links to the necroptosis pathway. CARD9 is a key signaling adaptor downstream Dectin-1 activation [37]. We found that siRNA-mediated knockdown of CARD9 in macrophages made the cells resistant to the Dectin-1 ligands-induced necroptosis, similar to siRNAmediated knockdown of Dectin-1 (Fig. 3e, f). CARD9 is essential for Dectin-1-induced NF-kB-dependent TNF production [5] and TNF is a strong inducer of necroptosis. To determine whether CARD9 mediated necroptosis through inducing TNF production, we knocked down CARD9 in $\mathrm{Tnf}^{-/-}$macrophages and found that CARD9 was still required for the Dectin-1 agonists-induced cell death in the $\mathrm{Tnf}^{-/}$macrophages (Fig. 3g). We also observed that silence of CARD9 in $T n f^{--}$macrophages impaired activation of MLKL induced by the Dectin-1 agonists (Fig. 3h, i). These results indicate that CARD9 may be directly involved in the activation of the necroptotic signaling cascade of RIPK1-RIPK3-MLKL in Dectin-1-induced necroptosis. Thus, we checked the potential interaction between RIPK1, RIPK3 and CARD9. We found that CARD9 interacted with RIPK1 and RIPK3 in overexpression system (Fig. 4a, b). To determine if these interactions are direct associated, we transcribed and translated CARD9, RIPK1 and RIPK3 in vitro and found that CARD9 directly interacted with RIPK1 but not with RIPK3 (Fig. 4c, d). To avoid the overexpression system, we immunoprecipitated the RIPK1 and RIPK3 complex by endogenous RIPK1 antibody and found endogenous RIPK1 associated with CARD9 and RIPK3 in the zymosan signal-dependent manner (Fig. 4e). Considering that RIPK1 kinase activity was required for the Dectin-1 ligands-induced activation of RIPK3 and MLKL (Fig. 2), these results indicate that CARD9 is the direct upstream signaling adaptor that links the Dectin-1 activation to the necroptotic signaling cascade (RIPK1-RIPK3MLKL) to induce necroptotic cell death. 

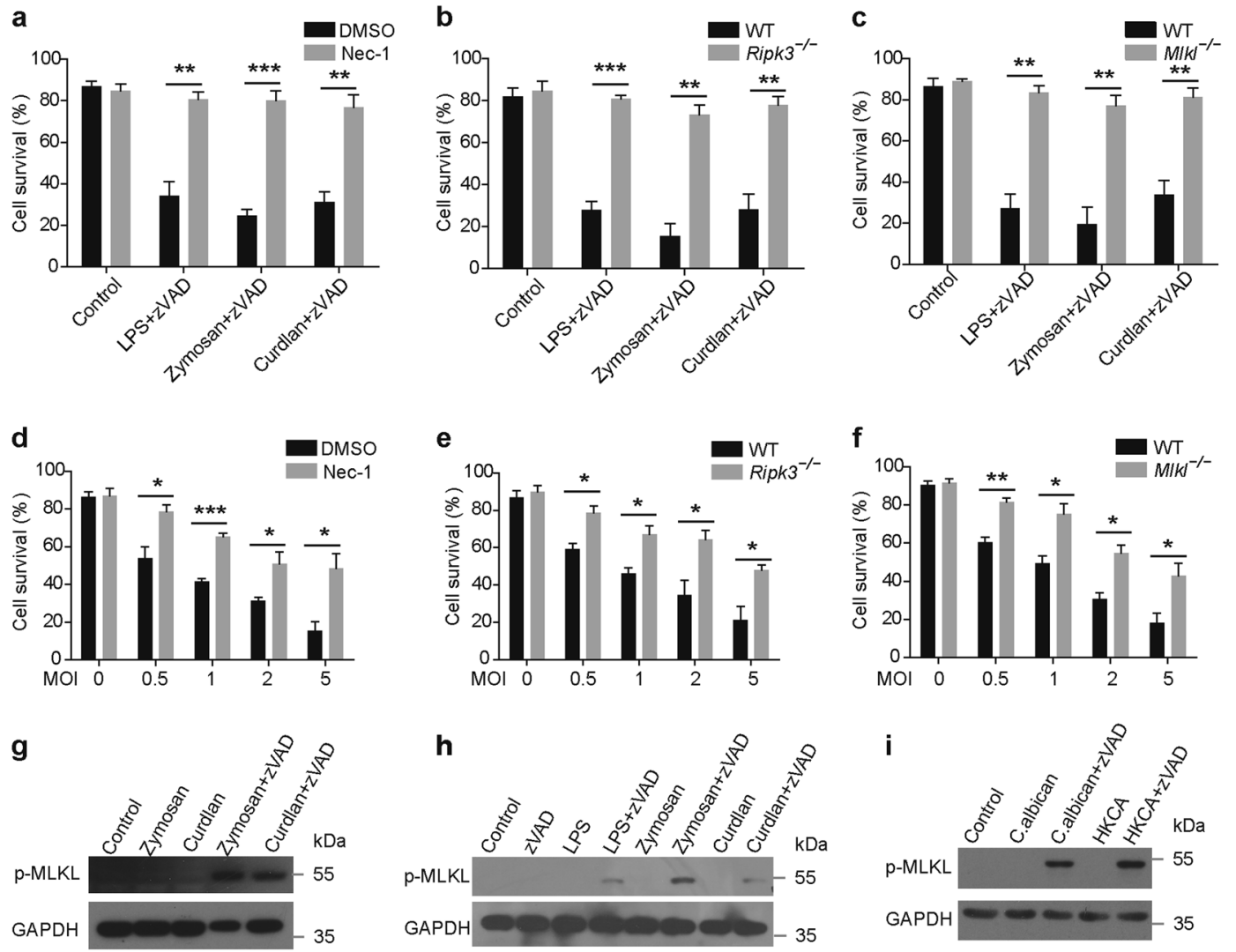

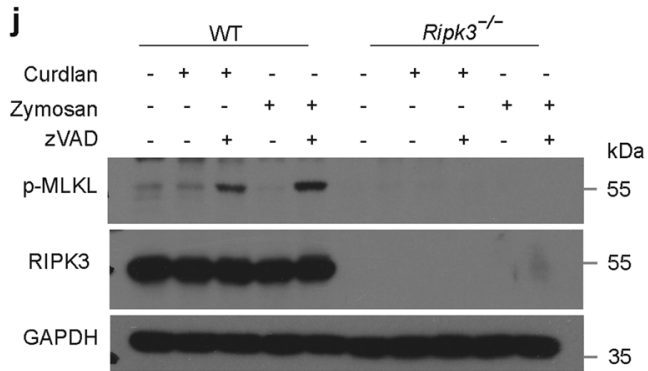

Fig. 2 RIPK1, RIPK3 and MLKL are required for fungus and its PAMPs-induced cell death. a Peritoneal macrophages were treated with the indicated ligands and $\mathrm{Nec}-1(50 \mu \mathrm{m} / \mathrm{ml})$ for $12 \mathrm{~h}$. Cell viability was determined by measuring the released LDH. b, c Peritoneal macrophages from wild-type, Ripk3 ${ }^{-/-}$mice (b) or $\mathrm{Mlkl}^{-/-}$mice (c) were treated with the indicated ligands and zVAD for $12 \mathrm{~h}$. Cell viability was determined by measuring released LDH. d Peritoneal macrophages were treated with different MOI of $C$. albicans and zVAD for $12 \mathrm{~h}$. Cell viability was determined by measuring released LDH. e, f Peritoneal macrophages from wild-type, $R i p k 3^{-/-}$mice (e) or $M l k l^{-1-}$ mice (f) were treated with different MOI of C. albicans and zVAD for $12 \mathrm{~h}$. Cell viability as determined by measuring released LDH. g Immunoblot analysis of p-MLKL from peritoneal

\section{Necroptosis protects host against Candida albicans infection in vivo}

While induction of necroptosis in vitro is characterized in the need to inhibit caspase- 8 activity, necroptosis has been k

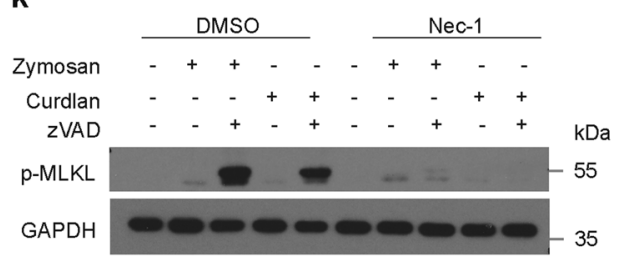

macrophages treated with zymosan or curdlan together with or without $\mathrm{zVAD}$ for $3 \mathrm{~h}$. h Immunoblot analysis of p-MLKL (S358) from THP1-derived macrophages treated with zymosan or curdlan together with or without $\mathrm{zVAD}$ for $3 \mathrm{~h}$. i Immunoblot analysis of pMLKL from peritoneal macrophages treated with $\mathrm{MOI}=100$ heat killed $C$. albicans $(\mathrm{HKCA})$ or $\mathrm{MOI}=5 \mathrm{C}$. albicans together with $\mathrm{zVAD}$ or not for 3 h. j, k Immunoblot analysis of p-MLKL from wild-type, Ripk $3^{-/}(\mathbf{j})$ or Nec-1 pretreated (k) peritoneal macrophages treated with zymosan or curdlan together with or without zVAD for $3 \mathrm{~h}$. Data are representative of three independent experiments. Error bars in (a-f) represent mean \pm SEM. $* p<0.05 ; * * p<0.01 ; * * * p<0.001$ by two-tailed test. See also Fig. S3

observed in the absence of caspase- 8 inhibition in vivo [38], indicating that apoptosis is somehow suppressed in in vivo situations. In the animal model of TNF-induced Lethal Systemic Inflammatory Response Syndrome (SIRS), necroptosis is crucial for the lethality of 
a

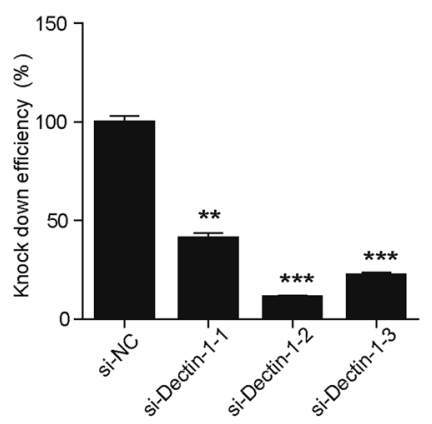

C

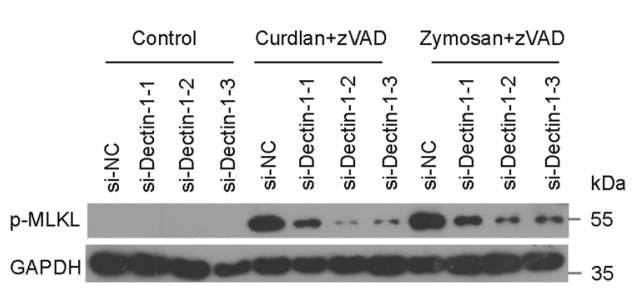

b

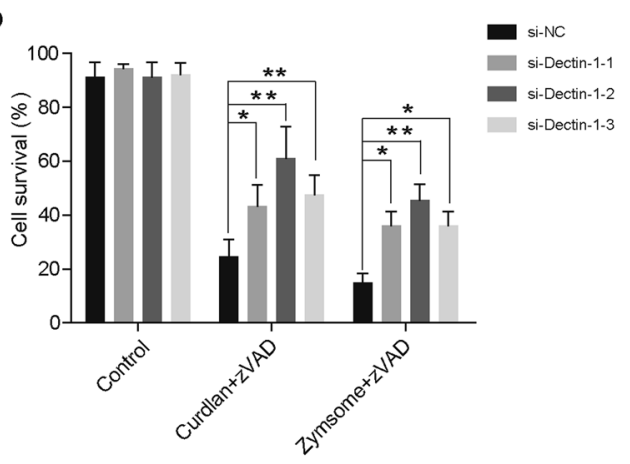

e

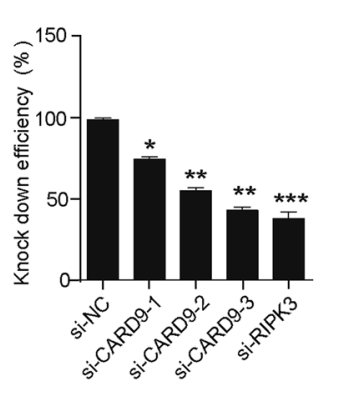

f

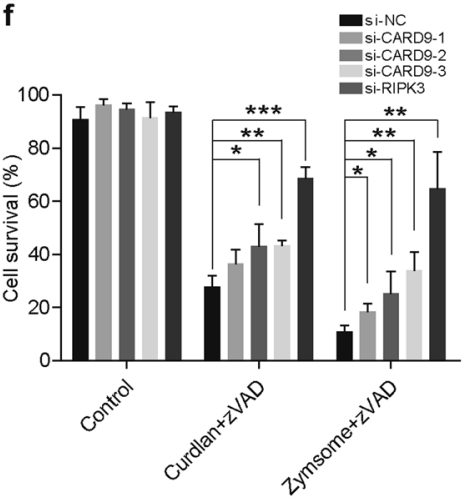

h
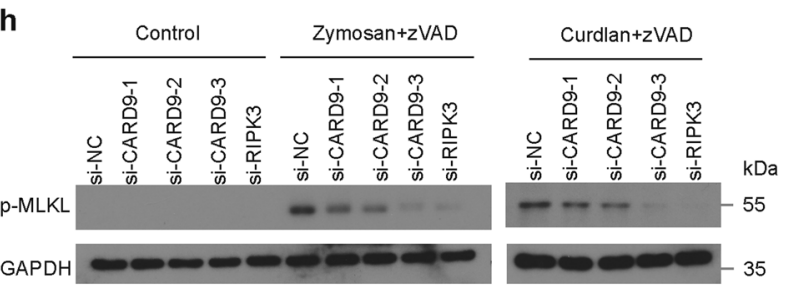

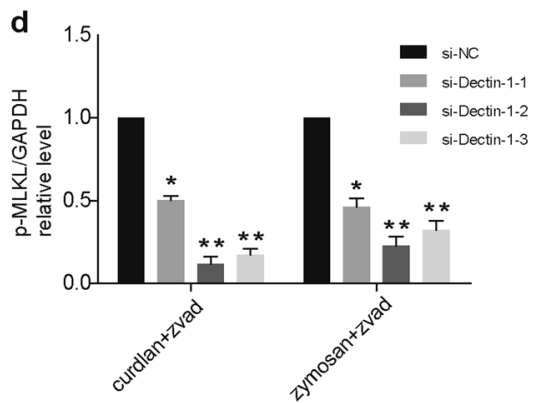

g

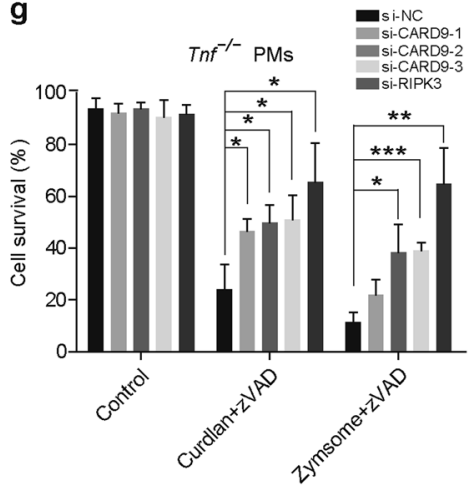

i

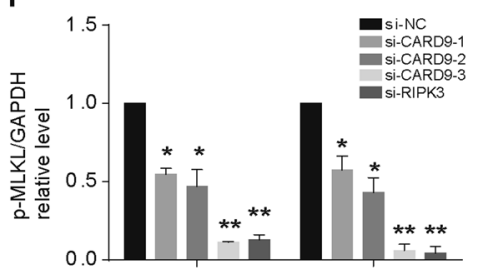

TNF-induced SIRS without caspase-8 inhibitor [39]. Therefore, these prompted us to examine whether Dectin1-induced necroptosis contributes to host defense $C$. albicans infection. To explore the potential role of necroptosis in fungal infection in vivo, we injected sublethal dose of C. albicans to wild-type and Ripk3 ${ }^{-/-}$mice and found that Ripk3 ${ }^{-/-}$mice showed more susceptibility to $C$. albicans infection compared with wild-type mice
(Fig. 5a). Consistently, fungal loads in kidneys, livers and spleens of Ripk $^{-/-}$mice were significantly increased compared with those of wild-type mice under the same low dose of fungal infection (Fig. 5b). Ripk $3^{-1-}$ mice also exhibited increased renal inflammation and higher $C$. albicans loads in the kidney (Fig. 5c, d). To further investigate the role of RIPK1-mediated necroptosis during C. albicans infection, mice were pretreated with RIPK1 
Fig. 3 Dectin-1 and CARD9 are required for fungus and its PAMPsinduced necroptotic cell death. a Peritoneal macrophages were transfected with scrambled siRNA oligos, Dectin-1 siRNA oligos for $60 \mathrm{~h}$. Knock-down efficiency was determined by detecting mRNA level through qPCR. b Peritoneal macrophages were transfected with the indicated siRNA oligos for $60 \mathrm{~h}$ and treated with zymosan or curdlan for $12 \mathrm{~h}$. Cell viability was determined by measuring released LDH. c Immunoblot analysis of p-MLKL from peritoneal macrophages transfected with the indicated siRNA oligos for $60 \mathrm{~h}$ and then treated with zymosan, curdlan and zVAD for $3 \mathrm{~h}$. d Densitometric quantification of p-MLKL/GAPDH relative level is shown from (c) . e Peritoneal macrophages were transfected with scrambled siRNA oligos, CARD9 siRNA oligos or RIPK3 siRNA oligos for $60 \mathrm{~h}$. Knock-down efficiency was determined by detecting mRNA level through qPCR. f Peritoneal macrophages were transfected with the indicated siRNA oligos for $60 \mathrm{~h}$ and treated with zymosan or curdlan for $12 \mathrm{~h}$. Cell viability was determined by measuring released LDH. g Peritoneal macrophages from $\mathrm{Tnf}^{-/}$mice were transfected with the indicated siRNA oligos for $60 \mathrm{~h}$ and treated with zymosan, curdlan and zVAD for $12 \mathrm{~h}$. Cell viability was determined by measuring released LDH. h Immunoblot analysis of p-MLKL from $\mathrm{Tnf}^{-/-}$peritoneal macrophages transfected with the indicated siRNA oligos for $60 \mathrm{~h}$ and then treated with zymosan, curdlan and zVAD for $3 \mathrm{~h}$. i Densitometric quantification of p-MLKL/GAPDH relative level is shown from (h). Data are representative of three independent experiments. Error bars in $(\mathbf{a}, \mathbf{b}, \mathbf{d}-\mathbf{g}, \mathbf{i})$ represent mean \pm SEM. $* p<0.05 ; * * p<0.01$; $* * * p<0.001$ by two-tailed test

inhibitor Nec-1 and followed by infection with sublethal dose of $C$. albicans. The mice pretreated with Nec-1 showed more susceptibility to $C$. albicans infection and had more fungal load in their tissues (Fig. 5e, f). Since RIPK1 and RIPK3 have also been reported to have necroptosis-independent function in inflammatory signaling pathway, the phenotypes from RIPK3 and RIPK1deficient mice may not reflect the function of necroptosis [40-42]. To determine the potential role of necroptosis in host defense against fungal infection, we infected $M l \mathrm{kl}^{-/-}$ mice with sublethal dose of $C$. albicans, and found that $M l k l^{-1-}$ mice showed more susceptibility to $C$. albicans infection compared to WT control mice (Fig. 5g, h). However, the susceptibility of $M l k l^{-/-}$mice to C. albicans infection was weaker than that of Ripk $3^{-/-}$mice (Fig. 5h). These in vivo results suggest that MLKL-dependent necroptosis is important for host defense against fungal infection while RIPK1 and RIPK3 also have MLKLindependent function in host defense against fungal infection. As necroptosis can promote inflammation in contrast to apoptosis, we analyzed the expression of inflammatory genes in kidney and found that the expression levels of Il6, GM-CSF and $I l 1 \beta$ in Ripk $3^{-/-}$mice were much higher than those in wild-type mice at day 5 (Fig. S5a). As the increased inflammatory genes expression might be due to more fungal loads in the deficient mice, we therefore analyzed the inflammatory gene expression in the early period of infection, and found that Ripk $^{-/-}$mice showed impaired proinflammatory cytokines production (Fig. S5b). Thus, the impaired inflammation in $R i p k 3^{-/-}$mice during the early period of infection likely contributed to its higher susceptibility of C. albicans infection.

\section{RIPK1 and RIPK3 potentiate Dectin-1-induced MLKL- independent inflammation}

As described above, Ripk $3^{-1-}$ mice showed more susceptibility to $C$. albicans infection compared with $M l k l^{-/-}$mice (Fig. 5h), suggesting that RIPK3 has other function independent of the MLKL-mediated cell death during fungal infection. It has been recently reported that RIPK1 and RIPK3 promote TLR3 and TLR4-induced TRIF-dependent inflammation in the necroptotic cell death-independent manner [43]. To investigate whether RIPK1 and RIPK3 similarly regulate Dectin-1-induced inflammation, we stimulated macrophages with zymosan in the presence or absence of $\mathrm{zVAD}$ and found that $\mathrm{zVAD}$ could strongly potentiate zymosan-induced proinflammatory cytokines (Il6, GM-CSF, KC) expression (Fig. 6a). Knocking-down caspase- 8 in macrophages also promoted zymosan-induced cytokines expression (Fig. S6a). To determine whether RIPK1 and RIPK3 are involved in the inflammatory response, we used Ripk $3^{-/-}$macrophages or Nec-1 inhibitor and found that the increase of inflammatory genes expression induced by the dectin-1 agonists together with zVAD was abolished in Ripk $3^{-/-}$macrophages or Nec-1-pretreated macrophages (Fig. 6b, c and S6b, S6c). Consistently, the increase of IL-6 protein level was blocked by RIPK3 deficiency and RIPK1 kinase inhibition (Fig. 6d, e). To assess whether MLKL is involved in the Dectin-1-induced inflammation, we stimulated $M l k l^{-1-}$ macrophages with the denctin-1 agonists together with $\mathrm{zVAD}$ and found MLKL was not required for the Dectin-1-induced inflammation as the increase of IL-6 protein level and the induction of inflammatory genes expression were comparable between wild-type and $\mathrm{Mlkl}^{-/-}$macrophages (Fig. 6f, g). Collectively, these data demonstrated that RIPK1 and RIPK3 potentiated Dectin-1-induced inflammation in a necroptotic cell death-independent manner.

\section{JNK and P38 are required for the Dectin-1-induced RIPK1 and RIPK3-mediated inflammation}

We showed above that RIPK1 and RIPK3 promoted Dectin1 -induced inflammation in an MLKL-mediated cell deathindependent manner (Fig. 6). To investigate how RIPK1 and RIPK3 potentiated the Dectin-1-induced inflammation, we examined the signaling pathway of NF- $\mathrm{B}$ and MAPKs and found that $\mathrm{zVAD}$-treated macrophages showed increased activation of P65 (NF- $\mathrm{BB}), \mathrm{JNK}$ and P38 but not ERK that were all induced by the Dentin-1 ligands zymosan and curdlan (Fig. 7a). We then utilized Nec-1 to inhibit 


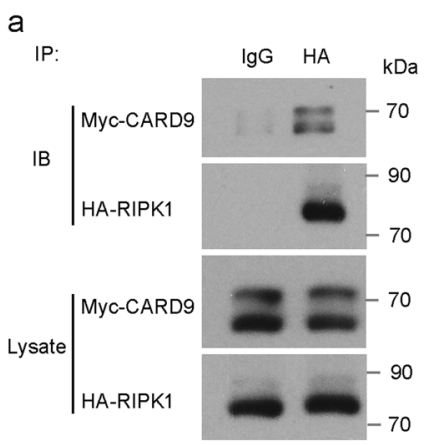

b

d

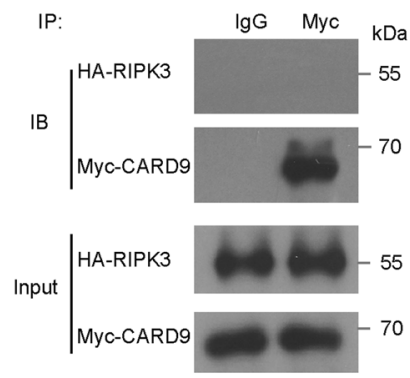

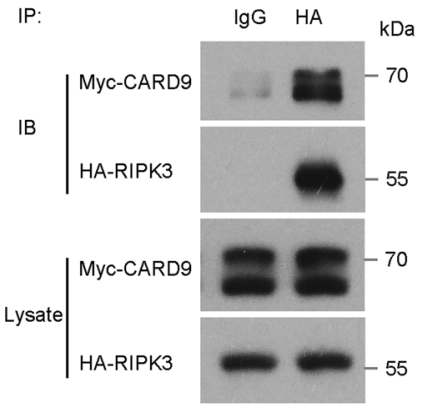

C
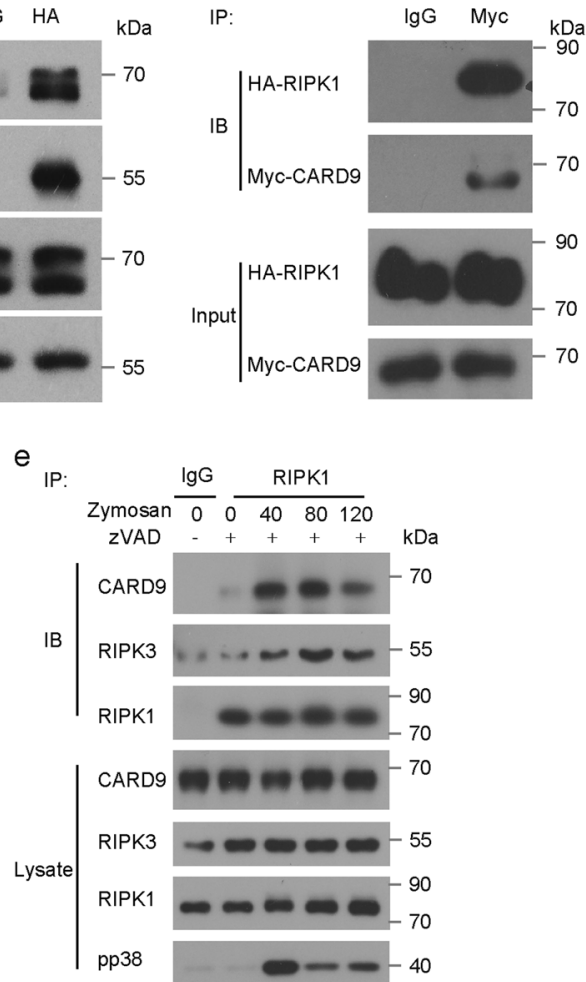

Fig. 4 CARD9 directly interacts with RIPK1 for necroptosis induction. a $293 \mathrm{~T}$ cells were transfected with plasmids expressing RIPK1-HA and CARD9-myc. Immunoprecipitation of RIPK1 by anti-HA antibody and immunoblot analysis of cell lysates and IP samples. b 293 T cells were transfected with plasmids expressing RIPK3-HA and CARD9-myc. Immunoprecipitation of RIPK3 by anti-HA antibody and immunoblot analysis of cell lysates and IP samples. c RIPK1-HA and CARD9-myc were expressed in in vitro transcription and translation system. Immunoprecipitation of CARD9 by anti-myc

RIPK1 kinase activity and found that Nec-1 severely suppressed the zVAD-enhanced activation of JNK and P38 but not P65 (Fig. 7b). Similarly, RIPK3 deficiency almost blocked the zVAD-enhanced Dectin-1-induced activation of JNK and P38 (Fig. 7c). However, under the same stimulation the activation level of JNK and P38 had no difference between $\mathrm{Mlkl}^{-/-}$macrophages and wild-type macrophages (Fig. 7d). These results suggest that RIPK1 and RIPK3specifically mediate the zVAD-enhanced Dectin-1-induced activation of JNK and P38 but not P65. To further investigate the role of JNK and P38 in the increased inflammation, we inhibited JNK and P38 activation respectively with their specific inhibitors. We observed that JNK inhibition dramatically reduced the expression of proinflammatory genes induced by zymosan and zVAD (Fig. 7e). P38 inhibition also decreased induction of the inflammatory genes by zymosan and zVAD (Fig. 7f). Together, our data demonstrated that JNK and P38 were responsible for the RIPK1 and RIPK3-mediated Dectin-1-induced inflammation under condition of apoptosis blockage by zVAD. antibody and immunoblot analysis of cell lysates and IP samples. d RIPK3-HA and CARD9-myc were expressed in in vitro transcription and translation system. Immunoprecipitation of CARD9 by antimyc antibody and immunoblot analysis of cell lysates and IP samples. e Immunoprecipitation of endogenous RIPK1 in peritoneal macrophages treated with zymosan and zVAD for different time points and immunoblot analysis of cell lysates and IP samples. Data are representative of three independent experiments

\section{Discussion}

Necroptosis is a recently identified type of programmed necrotic cell death. Some stimuli have been reported to trigger necroptosis process, including host-derived cytokines (TNF, FasL and interferons) as well as pathogen PAMPs (LPS and poly I:C) [44]. The kinases RIPK1 and RIPK3 are critical for the initiation and transduction of necroptosis signaling and MLKL is the downstream executor to disrupt cell membranes. In addition to the studies on PAMPs, some microbes have been reported to induce necroptosis, including virus and bacteria. However, whether fungus can induce necroptosis remains unknown. Here we showed that the fungus $C$. albicans as well as its PAMPs (zymosan and curdlan) triggered activation of the RIPK1-RIPK3-MLKL cascade and consequently necroptotic cell death in both human and mouse myeloid cells (THP1-derived macrophage, PM, BMDM and BMDC) under the conditions of apoptosis blockage or in the absence of caspase- 8 activity. We demonstrated that RIPK1 kinase 

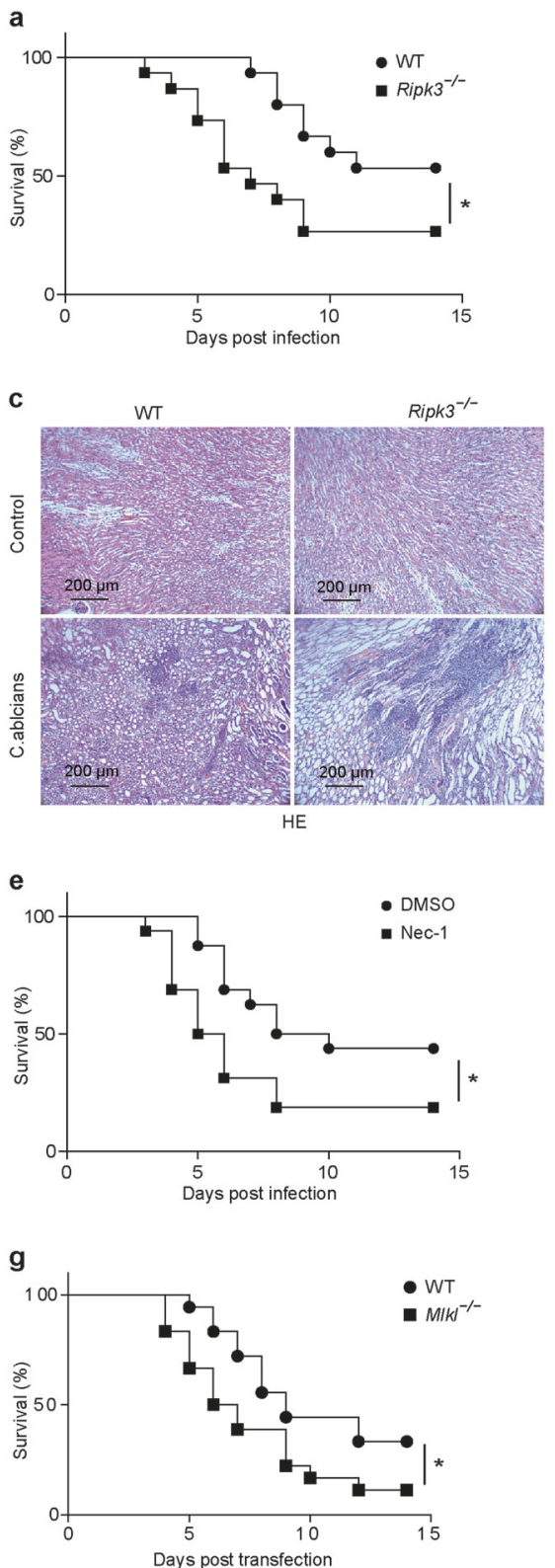

Fig. 5 Necroptosis protects hosts against Candida albicans infection. a Wild-type mice $(n=15)$ and Ripk $^{-1-}(n=15)$ mice were intravenously injected with $5 \times 10^{5} \mathrm{C}$. albicans per mice. Mouse survival was monitored and plotted. b Wild-type mice $(n=11)$ and $\operatorname{Ripk}^{-/-}(n=$ 11) mice were intravenously injected with $1 \times 10^{5}$ C. albicans per mice. After 5 days WT and Ripk $3^{-/-}$mice were sacrificed and kidneys, livers and spleens were collected to count fungal load. Each dot represents data from a single mouse. c HE staining of WT and Ripk3 ${ }_{--}^{-}$mice from (b). d PAS staining of WT and Ripk $3^{-/-}$mice from (b). e Wild-type mice were pretreated with DMSO $(n=16)$ or Nec-1 $(n=$ $16)$ and then infected with $5 \times 10^{5} C$. albicans per mice. Mouse survival was monitored and plotted. f Wild-type mice were pretreated with DMSO $(n=13)$ or Nec-1 $(n=13)$ and then infected with $1 \times 10^{5}$

activity, RIPK3 and MLKL were all required for the necroptosis induction by the fungus and its PAMPs. RIPK1 and RIPK3 were the upstream kinases for MLKL activation
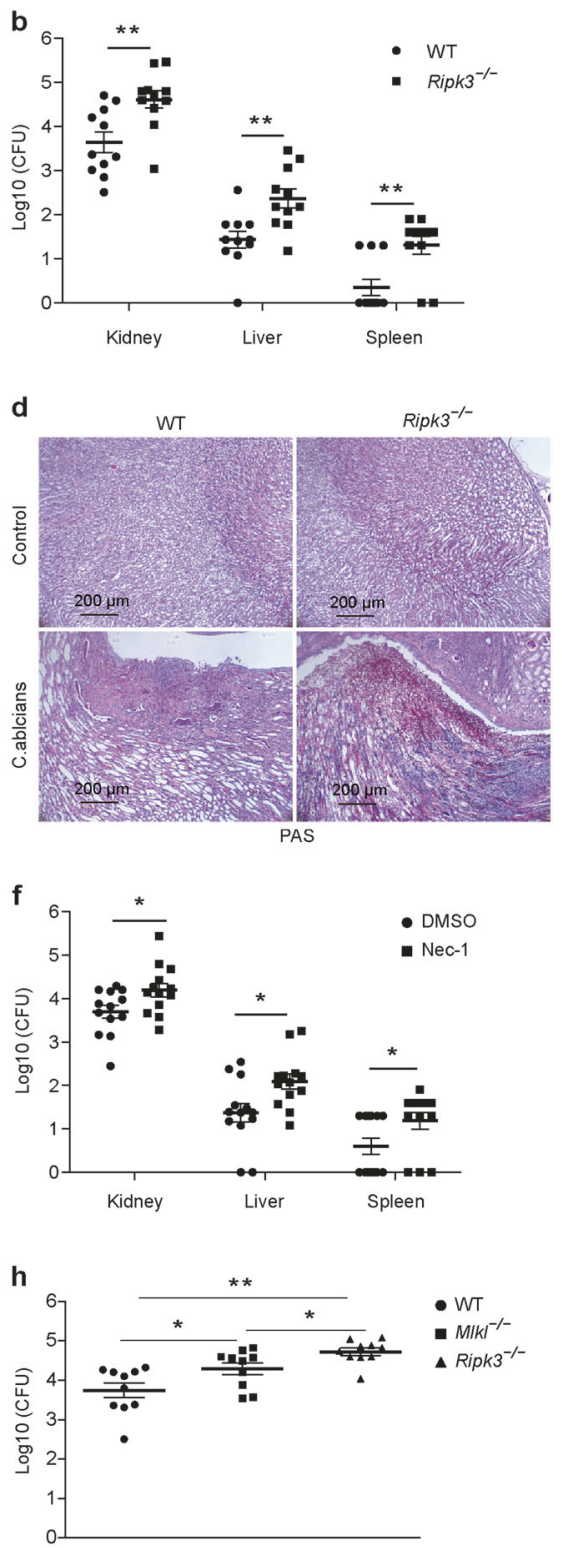

C. albicans per mice. Five days later, mice were sacrificed and kidneys, livers and spleens were collected to count fungal load. Each dot represents data from a single mouse. $\mathbf{g}$ Wild-type mice $(n=18)$ and $\mathrm{Mlkl}^{-1-}$ mice $(n=18)$ were intravenously injected with $5 \times 10^{5} \mathrm{C}$. albicans per mice. Mouse survival was monitored and plotted. $\mathbf{h}$ Wildtype mice $(n=10), \operatorname{Ripk}^{-/-}$mice $(n=10)$ and $\operatorname{Mlkl}^{-1-}(n=10)$ mice were intravenously injected with $1 \times 10^{5} \mathrm{C}$. albicans per mice. Five days later, mice were sacrificed and kidneys were collected to count fungal load. Each dot represents data from a single mouse. Data in (c) are representative of three independent experiments. Data in $(\mathbf{a}, \mathbf{b}$ and $\mathbf{e}-\mathbf{h}$ ) are compiled from two or three independent experiments. Error bars in $(\mathbf{a}, \mathbf{b}, \mathbf{e}-\mathbf{h})$ represent mean \pm SEM. $* p<0.05 ; * * p<0.01$ by two-tailed test

in the fungal PAMPs-triggered necroptosis process. We also found that Dectin-1 was the sensor for the fungal PAMPs to induce necroptotic cell death. We further demonstrated that 

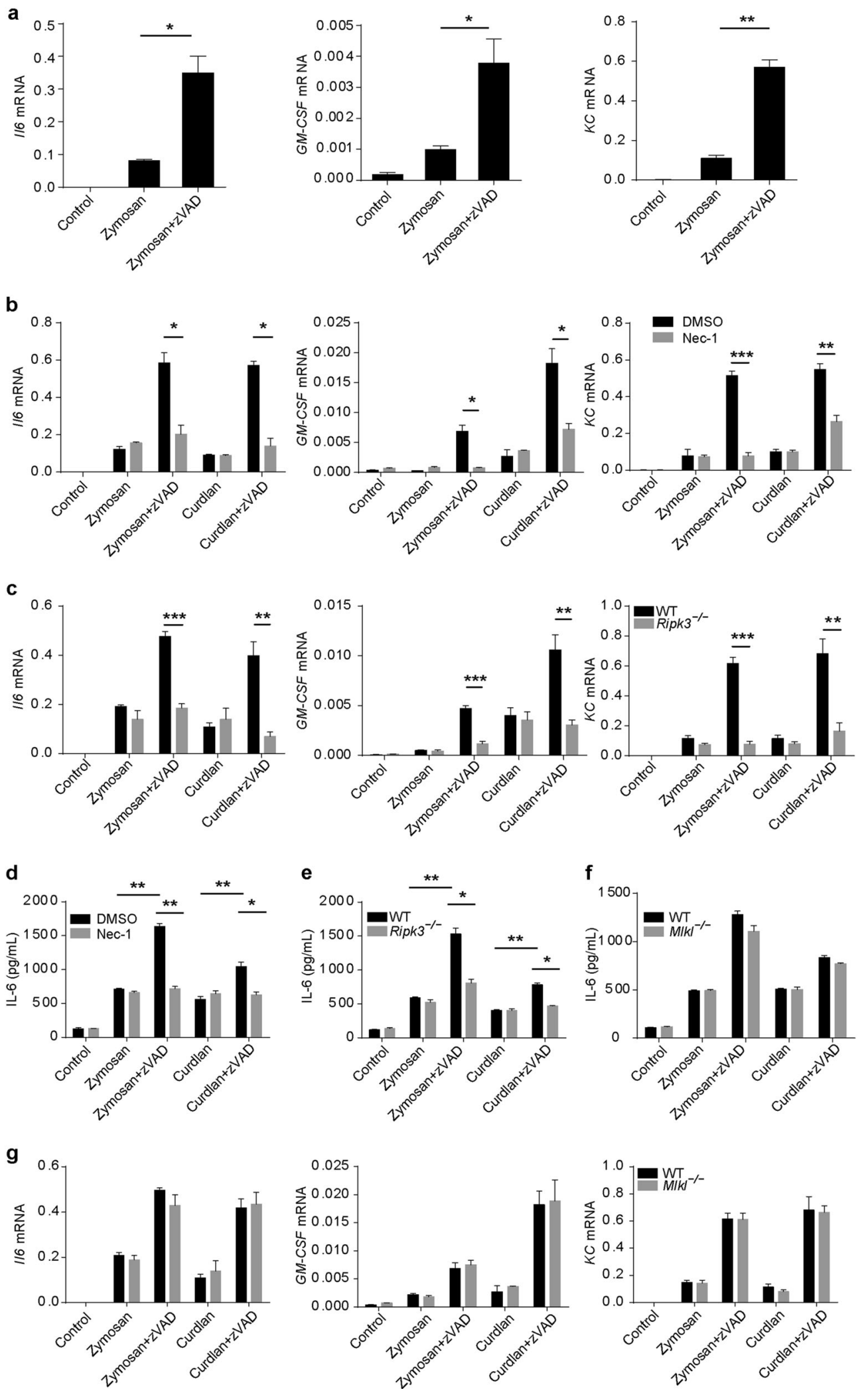

the fungus and its PAMPs-induced necroptosis was not dependent on autocrine TNF production by utilizing $\operatorname{Tnf}^{-/-}$ macrophages.
As Dectin-1 activation triggered RIPK1, RIPK3 and MLKL-dependent necroptosis in macrophages independent of autocrine TNF, we hypothesized that Dectin-1 signaling 
Fig. 6 Dectin-1-induced signaling triggers MLKL-independent but RIPK1 and RIPK3-dependent inflammatory response. a qPCR analysis of $I l 6, G M-C S F$ and $K C$ mRNA in wild-type peritoneal macrophages treated with zymosan and zVAD for $3 \mathrm{~h}$. b qPCR analysis of Il6, GM-CSF and $K C$ mRNA in wild-type peritoneal macrophages treated with the indicated ligands and zVAD for $3 \mathrm{~h}$. $\mathbf{c}$ qPCR analysis of Il6, GM-CSF and KC mRNA in wild-type or Ripk $3^{-/-}$peritoneal macrophages treated with the indicated ligands and zVAD for $3 \mathrm{~h} . \mathbf{d}-\mathbf{f}$ Elisa analysis of IL-6 in Nec-1 pretreated peritoneal macrophages (d), $\mathrm{Ripk3}^{-/-}$peritoneal macrophages (e) or $\mathrm{Mlkl}^{-/-}$peritoneal macrophages (f) treated with the indicated ligands and zVAD. g qPCR analysis of $I l 6, G M-C S F$ and $K C$ mRNA in wild-type or $M l k l^{-/-}$ peritoneal macrophages treated with the indicated ligands and zVAD for $3 \mathrm{~h}$. Data are representative of three independent experiments. Error bars in (a-g) represent mean \pm SEM. $* p<0.05$; $* * p<0.01 ; * * * p<$ 0.001 by two-tailed test. See also Fig. S6

may directly activate the necroptotic pathway of RIPK1, RIPK3 and MLKL. Interestingly, we found that CARD9, the key adaptor of Dectin-1 signaling, interacted with RIPK1 both in the overexpression system and in the endogenous system in ligand-stimulation-dependent manner. Consistently, knockdown of CARD9 in wild-type or $\mathrm{Tnf}^{-/-}$macrophages greatly decreased the activation of MLKL as well as the necroptotic cell death induction by the Dectin-1 ligands. These data suggest that CARD9 links upstream Dectin-1 signaling to the necroptotic cascade RIPK1-RIPK3-MLKL to perform necroptotic cell death under fungal infection.

Although its potential pathophysiological roles are still largely undefined, necroptosis has recently been shown to participate in host defense against viral infections. RIPK3 and MLKL mediate the virus-induced necroptosis and the deficient mice showed susceptibility to HSV-1 and influenza virus infections [9]. Here, for the first time we demonstrate that fungus as well as its PAMPs can induce necroptosis through RIPK1, RIPK3 and MLKL in myeloid cells. We further found that both $\mathrm{Ripk}^{-/-}$and $M l k l^{-/-}$mice showed higher fungal burden and lethality compared to their representative WT control mice. Similarly, RIPK1 kinase inhibition by Nec-1 made mice more susceptible to $C$. albicans infection. These data suggest that fungus-induced necroptosis is important for host defense against fungal infection. Considering necroptosis can result in release of cellular contents like damage-associated molecular-pattern (DAMP) molecules that can stimulate macrophages and dendritic cells to induce production of cytokines and chemokines [45], Dectin-1-induced necroptosis may thus function to amplify the local inflammation to recruit more neutrophils and macrophages to control $C$. albicans infection. In the early period of fungal infection, we observed impaired inflammatory response in Ripk $3^{-/-}$mice in vivo, which could be the reason of the susceptibility in Ripk $3^{-1-}$ mice.

When we compared the phenotypes of RIPK3-deficent mice with those of $\mathrm{Mlkl}^{-/-}$mice, we found that RIPK3deficent mice were more susceptible to $C$. albicans infection, suggesting that RIPK3 has MLKL-independent function. RIPK3 has been known to have necroptosis-independent function in inflammasome activation induced by virus [24]. In addition, RIPK3 is also reported to be required for West Nile virus-induced neuroinflammation in a cell deathindependent manner [46]. It has been well known that RIPK1 is vital for TNF signaling and TLR signaling as a scaffold protein in kinase independent manner [47]. Recently, it is reported that RIPK1 and RIPK3 potentiate TRIF-dependent proinflammatory cytokines release through ERK signaling [43]. Similarly, we found that RIPK1 and RIPK3 promoted the Dectin-1 ligands-induced expression of inflammatory genes which was MLKL-independent under the condition of apoptosis blockage. However, we found that JNK and P38 pathways instead of ERK signaling mediated the promoting effects of RIPK1 and RIPK3 on the Dectin-1induced inflammation. Although the detailed mechanisms remain to be further explored, our data suggest that RIPK1 and RIPK3 utilize differential signaling pathways to promote cell death-independent inflammation driven by different kinds of stimuli. Together, we demonstrate that fungus and its Dectin-1 ligands activate the necroptotic signaling cascade of RIPK1-RIPK3-MLKL through CARD9 in myeloid cells and that both the MLKL-dependent necroptotic cell death and the RIPK1 and RIPK3-mediated MLKL-independent inflammation contribute to hose defense against fungal infection. RIPK1, RIPK3 and MLKL are therefore potential new targets for developing antifungal strategies.

\section{Materials and methods}

\section{Mice}

Wild-type C57BL/6J mice, MRL/lpr mice, ICR mice were purchased from Slac Laboratory Animal Center, Chinese Academy of Sciences. Ripk3 ${ }^{-/-}$mice (on the C57BL/6 background) were generated from Ripk3tml(KOMP)Wtsi sperm purchased from KOMP Repository. $M l k l^{-/-}$mice (on the C57BL/6 background) were provided by Dr. Haibing Zhang (Shanghai Institute for Biological Sciences, Chinese Academy of Sciences). $\mathrm{Tnf}^{-1-}$ mice (strain

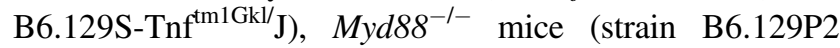
(SJL)-Myd88 $8^{\mathrm{tm} 1.1 \mathrm{Defr}} / \mathrm{J}$ ), Trif ${ }^{\text {PS2 } 2}$ mice (strain C57BL/6JTicam $1^{\mathrm{Lps} 2} / \mathrm{J}$ ) were purchased from the Jackson Laboratory. All mice were maintained in specific pathogen-free conditions at the Institute of Health Sciences. Six- to 10-week-old and gender-matched mice were used in all experiments. All animal experiments were performed according to the guidelines for the care and use of laboratory animals and are approved by the institutional biomedical research ethics committee of the Shanghai Institutes for Biological Sciences (Chinese Academy of Sciences). 
Fig. 7 JNK and P38 are required for Dectin-1-induced RIPK1 and RIPK3-mediated inflammatory response. a Immunoblot analysis of phosphorylated P65, ERK, JNK, P38 and GAPDH in peritoneal macrophages treated with the indicated ligands and zVAD for $3 \mathrm{~h}$. b Immunoblot analysis of phosphorylated P65, ERK, JNK, P38 and GAPDH in peritoneal macrophages pretreated with or without $\mathrm{Nec}-1$ and followed by the indicated ligands treatment for $3 \mathrm{~h}$. c Immunoblot analysis of phosphorylated P65, ERK, JNK, P38 and GAPDH in wild-type and Ripk $3^{-/-}$peritoneal macrophages treated with the indicated ligands and $\mathrm{zVAD}$ for $3 \mathrm{~h}$. d Immunoblot analysis of phosphorylated ERK, JNK, P38 and GAPDH in wild-type and $\mathrm{Mlkl}^{-/-}$peritoneal macrophages treated with the indicated ligands and $\mathrm{zVAD}$ for $3 \mathrm{~h}$. e, $\mathbf{f}$ qPCR analysis of $I l 6$ and $K C$ mRNA in peritoneal macrophages pretreated with JNK inhibitor SP600125 (e) or P38 inhibitor SB203580 (f) and then treated with the indicated ligands and zVAD for $3 \mathrm{~h}$. Data are representative of three independent experiments. Error bars in $(\mathbf{e}, \mathbf{f})$ represent mean \pm SEM. $* p<0.05 ; * * p<0.01$ by two-tailed test a

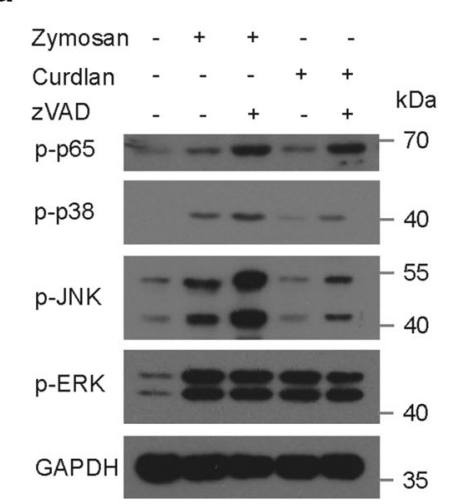

C

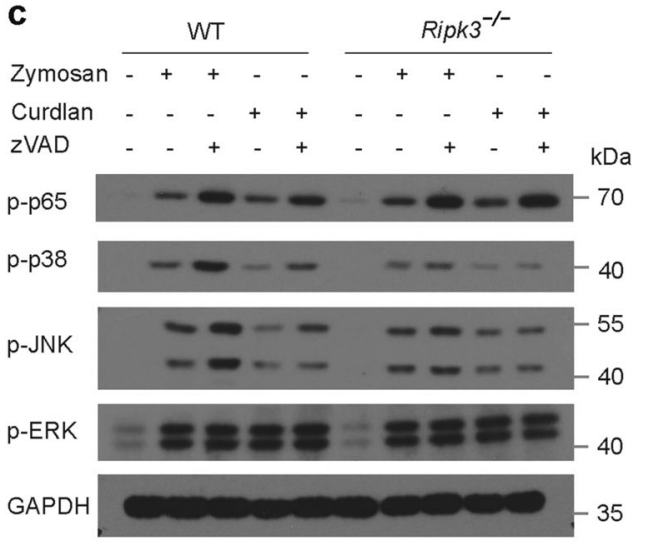

b

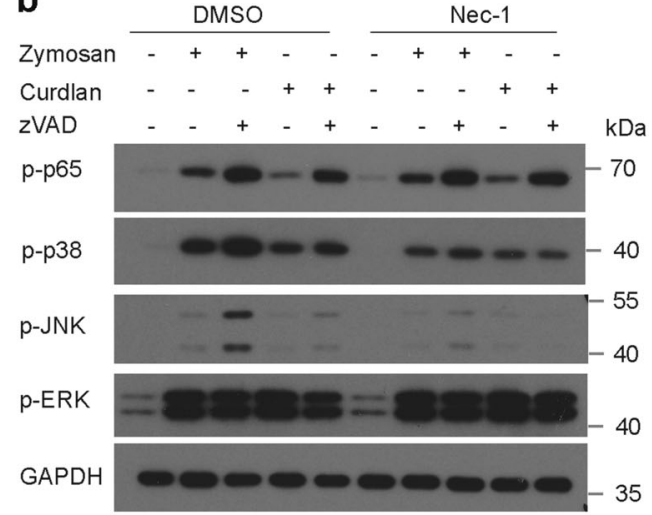

d
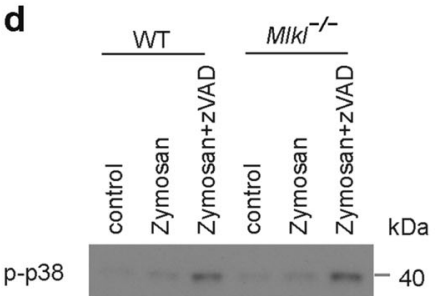

p-JNK

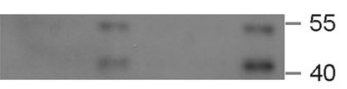

p-ERK

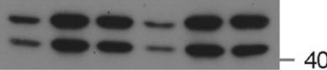

GAPDH

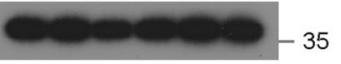

e
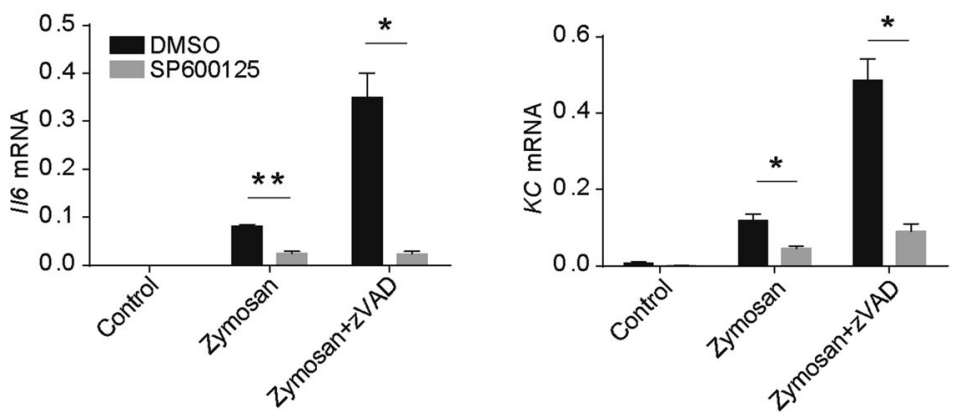

f

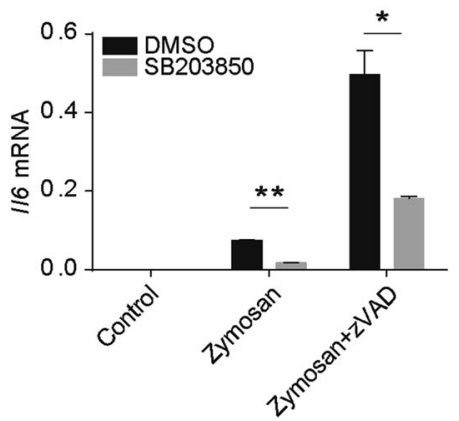

\section{Cells}

HEK293T cells and mouse macrophage line J774a.1 cells were cultured in high glucose Dulbecco's modified Eagle's medium (DMEM) supplemented with $10 \%$ FBS, $100 \mathrm{U} / \mathrm{ml}$ penicillin and $100 \mu \mathrm{g} / \mathrm{ml}$ streptomycin. THP-1 cells were cultured in RPMI 1640 medium containing 10\% FBS, $100 \mathrm{U} /$ $\mathrm{ml}$ penicillin, $100 \mu \mathrm{g} / \mathrm{ml}$ streptomycin, THP-1-derived 
macrophages were induced by $50 \mathrm{ng} / \mathrm{ml}$ phorbol 12-myristate-13-acetate (PMA) overnight. Peritoneal macrophages, derived from wild-type or the indicated deficient mice injected with $4 \%$ thioglycolate medium for 3 days, were cultured in high glucose DMEM with $10 \%$ FBS, $100 \mathrm{U} / \mathrm{ml}$ penicillin and $100 \mu \mathrm{g} / \mathrm{ml}$ streptomycin. Bone marrow-derived macrophages from C57BL/6 mice were derived by incubating murine bone marrow with DMEM containing 30\% L929 conditioned medium supplemented with $10 \%$ FBS, $100 \mathrm{U} / \mathrm{ml}$ penicillin and $100 \mu \mathrm{g} / \mathrm{ml}$ streptomycin. After 6 days, the differentiated cells were used for experiments. Bone marrowderived dendritic cells from C57BL/6 mice were generated by incubating murine bone marrow with RPMI 1640 medium containing $10 \% \mathrm{FBS}, 100 \mathrm{U} / \mathrm{ml}$ penicillin, $100 \mu \mathrm{g} / \mathrm{ml}$ streptomycin and $20 \mathrm{ng} / \mathrm{ml} \mathrm{mGM-CSF}$. After 9 days, the differentiated cells were used for experiments.

\section{Mouse model of systemic candidiasis}

Candida albicans SC5314 strains were routinely grown in YPD medium overnight at $30^{\circ} \mathrm{C}$. C. albicans were centrifuged, re-suspended with sterile PBS and counted with hematocytometer. To monitor the survival of wild-type and the indicated deficient mice, $5 \times 10^{5}$ C. albicans were injected to the indicated mice via tail veins. For the inhibition of RIPK1 in vivo, $125 \mu \mathrm{g}$ Nec-1 was injected intravenously to mice $30 \mathrm{~min}$ before $C$. albicans infection. To determine fungal load, mice were given at the dose of $1 \times 10^{5}$ C. albicans via tail veins. After 5 days, organs were aseptically removed and homogenized in sterile PBS. Homogenized organs were diluted with sterile PBS and plated on YPD agar plates. Colony-forming units (CFUs) were counted after $24 \mathrm{~h}$ on YPDA agar plates at $30^{\circ} \mathrm{C}$.

\section{In vitro stimulation of cells}

J774.1 cells, BMDMs, BMDCs and PMs, THP-1-derived macrophages were seeded in 96-well or 24-well plates. Cells were pretreated with the indicated inhibitors $(20 \mu \mathrm{M} \mathrm{z-VAD-}$ FMK, $20 \mu \mathrm{M}$ SP600125, $10 \mu \mathrm{M}$ SB203580) for $30 \mathrm{~min}$. After that, cells were stimulated with the indicated ligands ( $25 \mathrm{ng} / \mathrm{ml}$ LPS, $100 \mu \mathrm{g} / \mathrm{ml}$ zymosan, $100 \mu \mathrm{g} / \mathrm{ml}$ curdlan, $1 \mu \mathrm{g} / \mathrm{ml}$ pam3csk4, MOI $=100 \mathrm{HKCA}$ ) for the indicated time. HKCA were produced by boiling $C$. albicans at $100{ }^{\circ} \mathrm{C}$ for $30 \mathrm{~min}$ and re-suspended with sterile PBS.

\section{Cell viability assays}

Cell viability was determined by detecting released lactate dehydrogenase (LDH) or PI (propidium iodide)-positive cells. For LDH release assay, $1 \times 10^{5}$ cells were seeded in 96-well plates. Before stimulation, medium was changed to OPTI MEM. Cells were stimulated with the indicated ligands or chemicals for the indicated time. Supernatant was collected to detect released LDH with CytoTox96 LDH release kit (Promega). Cell viability was determined by comparing released $\mathrm{LDH}$ to the total released $\mathrm{LDH}$ in the cell lysate. For PI staining, PMs were seeded in 24-well plates at the density of $5 \times 10^{5}$ cells. The cells were treated with the indicated ligands with or without zVAD for $12 \mathrm{~h}$. The cells were collected with EDTA (Ethylenediaminetetraacetic acid) -Tripsin, washed in PBS, and then stained with $2.5 \mu \mathrm{g} / \mathrm{ml}$ PI in PBS for $20 \mathrm{~min}$. After that, the cells were centrifuged and washed in PBS. The level of PIpositive cells was analyzed using a flow cytometer (BD Biosciences).

\section{siRNA knockdown in peritoneal macrophages}

Peritoneal macrophages and J774.1 cells were seeded in 96-well plates to detect cell viability or 24-well plates to harvest proteins or RNA. To knock down the gene expression of RIPK3, MLKL, caspase8, Tnfsf10 or CARD9, cells were transfected with Scrambled siRNA (control) or RIPK3 siRNA (5'GCUCUCGUCUUCAA CAACU3'), MLKL siRNA (5'GAACCUGCCCG AUGA

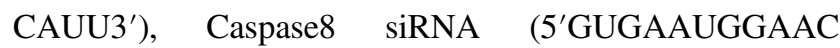
CUGGUAUA3'), CARD9- 1 siRNA (5'GCAAGGACGA GAACUAUGA3'), CARD9-2 siRNA (5'GGGUAAGC UAGACAGGAAU3'), CARD9-3 siRNA (5'GACAAA GAUAAGCUUCGAA3'), Dectin-1-1 siRNA (5'GGGAG GAUGGAUCAGCAUU3'), Dectin-1-2 siRNA (5'GC UGUUACCUAUUUAGCUU3'), Dectin-1-3 siRNA (5' GCCCAUUGCAGUGGGUU UA3'), Tnfsf10-1 siRNA (5' AAGAGGUG ACUUUGAGAACCUUU3'), Tnfsf10-2 siRNA (5'AAGCAGCUAAGUACUCCUCCCUU3'). The cells were incubated with transfectin mixture for $60 \mathrm{~h}$, and then treated with the indicated compounds for the indicated time. Knockdown efficiency was determined by detecting protein level or mRNA level.

\section{Immunoprecipitation and immunoblot analysis}

Immunoblot and immunoprecipitation were performed according to the following protocols. Briefly, the cells were lysed with lysis buffer (50 mM Tris- $\mathrm{HCl}$ (pH 7.5), $150 \mathrm{mM}$ $\mathrm{NaCl}, 1.0 \%$ Triton $\mathrm{X}-100,1 \mathrm{mM}$ EDTA and $10 \%$ glycerol containing protease inhibitors) for $30 \mathrm{~min}$. Cell lysates were collected by centrifugation at $13,000 \mathrm{rpm}$ at $4{ }^{\circ} \mathrm{C}$ for $30 \mathrm{~min}$. The cell lysates were incubated with anti-CARD9 antibody (sc-374569) from Santa Cruz and rotated overnight at $4{ }^{\circ} \mathrm{C}$. $15 \mu \mathrm{l}$ protein $\mathrm{A}$ beads were then added into the cell lysates and rotated for $3 \mathrm{~h}$ at $4{ }^{\circ} \mathrm{C}$. Beads were washed in lysis buffer for three times and then boiled in $20 \mu \mathrm{l} 1 \times$ SDS loading buffer for $10 \mathrm{~min}$. Protein samples were loaded on SDS-PAGE gels followed by electroblotting onto PVDF 
membranes. To determine protein expression level, immunoblot was performed using the following antibodies: antiRIPK3 (2283) from Pro Sci; anti-pMLKL S345 (ab196436) anti-pMLKL S358 (ab187091) from Abcam; anti-RIPK1 (610459) from BD Biosciences; anti-HA (MMS-101R) from Covance; anti-M2 (F1804) from Sigma; anti-myc, anti-pp65 (3033S), anti-pp38 (9211S) and anti-pJNK (9251) from Cell Signaling, anti-GAPDH, anti-CARD9 (sc-374569), anti-Caspase-1 P10 (sc-514) from Santa Cruz.

\section{RNA extraction and real-time PCR}

RNA of cells or tissues was extracted by the TRIzol reagent according to the manufacturer's protocols. For real-time PCR analysis, RNA was retro-transcribed by using PrimeScript RT reagent kit (TaKaR). Real-time PCR was performed using SYBR Premix ExTaq kit (TaKaRa) on ABI PRISM 7900 Sequence Detection System (Applied Biosystems). Targeted gene transcription was normalized to the level of housekeeping gene rpll3a. Primer sequences were listed in Table S1.

\section{Histology}

Kidneys from infected mice or control mice were fixed with $4 \%$ paraformaldehyde for at least 2 days. The fixed kidneys were embedded into paraffin and sliced into $5 \mu \mathrm{m}$ sections. Five micrometer sections were stained with H\&E (Hematoxylin-eosin staining) or PAS (Periodic Acid-Schiff stain) according to the standard procedures. Images were obtained with a Leica FDM2500 microscope.

\section{IL-6 cytokine measurement}

Peritoneal macrophages were treated with the indicated ligands for $12 \mathrm{~h}$ and culture supernatant was collected to measure IL-6 cytokine level using IL-6 Elisa kit according to the manufacturer's protocol.

\section{In vitro transcription and translation}

$\mathrm{TnT}^{\circledR}$ T7 Quick Coupled Transcription/Translation System from Promega was used according to the manufacturer's introduction.

\section{Quantification and statistical analysis}

Statistical analysis was performed using GraphPad Prism (GraphPad Software). Statistical significance between treated groups and their paired controls was determined using two-tailed standard $t$ tests. The differences of mice survival were analyzed by log-rank test. Statistical significance was defined as $p<0.05 . * p<0.05, * * p<0.01, * * * p<0.001$.
Acknowledgements This work was supported by grants from the National Natural Science Foundation of China (91842306, 81430036, 81830018, 91429307, and 91542119), National Key R\&D Program of China (2018YFA0507402) and the Strategic Priority Research Program of the Chinese Academy of Sciences (Grant No. XDB19000000).

Author contributions YQ and MC designed the experiments and wrote the manuscript; MC, ZW and Q.Lou conducted the experiments and analyzed the data. WL, JZ, YZ, YY, Q.Li, ML, and QZ helped with experiments. HZ provided $M l k l^{-/-}$mice. YQ supervised the study.

\section{Compliance with ethical standards}

Conflict of interest The authors declare that they have no conflict of interest.

Publisher's note: Springer Nature remains neutral with regard to jurisdictional claims in published maps and institutional affiliations.

\section{References}

1. Bleriot $\mathrm{C}$, Lecuit $\mathrm{M}$. The interplay between regulated necrosis and bacterial infection. Cell Mol Life Sci. 2016;73:2369-78.

2. Brown GD, Denning DW, Gow NA, Levitz SM, Netea MG, White TC. Hidden killers: human fungal infections. Sci Trans Med. 2012;4:165rv113-165rv113.

3. Netea MG, Joosten LA, Van Der Meer JW, Kullberg B-J, Van De, Veerdonk FL. Immune defence against Candida fungal infections. Nat Rev Immunol. 2015;15:630.

4. Geijtenbeek TB, Gringhuis SI. Signalling through C-type lectin receptors: shaping immune responses. Nat Rev Immunol. 2009;9:465.

5. Gross O, Gewies A, Finger K, Schäfer M, Sparwasser T, Peschel $\mathrm{C}$, et al. Card9 controls a non-TLR signalling pathway for innate anti-fungal immunity. Nature. 2006;442:651.

6. Gringhuis SI, Den Dunnen J, Litjens M, Van Der Vlist M, Wevers B, Bruijns SC, et al. Dectin-1 directs T helper cell differentiation by controlling noncanonical NF- $\mathrm{KB}$ activation through Raf-1 and Syk. Nat Immunol. 2009;10:203-13.

7. Geijtenbeek TB, Gringhuis SI. C-type lectin receptors in the control of $\mathrm{T}$ helper cell differentiation. Nat Rev Immunol. 2016;16:433-49.

8. Gringhuis SI, Kaptein TM, Wevers BA, Theelen B, Van Der Vlist M, Boekhout T, et al. Dectin-1 is an extracellular pathogen sensor for the induction and processing of IL-1 [beta] via a noncanonical caspase-8 inflammasome. Nat Immunol. 2012;13:246-54.

9. Weinlich R, Oberst A, Beere HM, Green DR. Necroptosis in development, inflammation and disease. Nat Rev Mol Cell Biol. 2017;18:127-36.

10. Oeckinghaus A, Hayden MS, Ghosh S. Crosstalk in NF-[kappa] B signaling pathways. Nat Immunol. 2011;12:695-708.

11. Cho Y, Challa S, Moquin D, Genga R, Ray TD, Guildford M, et al. Phosphorylation-driven assembly of the RIP1-RIP3 complex regulates programmed necrosis and virus-induced inflammation. Cell. 2009;137:1112-23.

12. Zhang D-W, Shao J, Lin J, Zhang N, Lu B-J, Lin S-C, et al. RIP3, an energy metabolism regulator that switches TNF-induced cell death from apoptosis to necrosis. Science. 2009;325:332-6.

13. He S, Wang L, Miao L, Wang T, Du F, Zhao L, et al. Receptor interacting protein kinase- 3 determines cellular necrotic response to TNF- $\alpha$. Cell. 2009;137:1100-11. 
14. Degterev A, Hitomi J, Germscheid M, Ch'en IL, Korkina O, Teng $\mathrm{X}$, et al. Identification of RIP1 kinase as a specific cellular target of necrostatins. Nat Chem Biol. 2008;4:313-21.

15. Kaiser WJ, Upton JW, Long AB, Livingston-Rosanoff D, Daley LP, Hakem R, et al. RIP3 mediates the embryonic lethality of caspase-8-deficient mice. Nature. 2011;471:368.

16. Newton K, Dugger DL, Wickliffe KE, Kapoor N, de Almagro MC, Vucic D, et al. Activity of protein kinase RIPK3 determines whether cells die by necroptosis or apoptosis. Science. 2014;343:1357-60.

17. Sun L, Wang H, Wang Z, He S, Chen S, Liao D, et al. Mixed lineage kinase domain-like protein mediates necrosis signaling downstream of RIP3 kinase. Cell. 2012;148:213-27.

18. Wang H, Sun L, Su L, Rizo J, Liu L, Wang L-F, et al. Mixed lineage kinase domain-like protein MLKL causes necrotic membrane disruption upon phosphorylation by RIP3. Molecular Cell. 2014;54:133-46.

19. Cai Z, Jitkaew S, Zhao J, Chiang H-C, Choksi S, Liu J, et al. Plasma membrane translocation of trimerized MLKL protein is required for TNF-induced necroptosis. Nat Cell Biol. 2014;16:55.

20. He S, Liang Y, Shao F, Wang X. Toll-like receptors activate programmed necrosis in macrophages through a receptorinteracting kinase-3-mediated pathway. Proc Natl Acad Sci USA. 2011;108:20054-9.

21. Thapa RJ, Nogusa S, Chen P, Maki JL, Lerro A, Andrake M, et al. Interferon-induced RIP1/RIP3-mediated necrosis requires PKR and is licensed by FADD and caspases. Proc Natl Acad Sci USA. 2013;110:E3109-E3118.

22. Dillon CP, Weinlich R, Rodriguez DA, Cripps JG, Quarato G, Gurung P, et al. RIPK1 blocks early postnatal lethality mediated by caspase- 8 and RIPK3. Cell. 2014;157:1189-202.

23. McComb S, Cessford E, Alturki NA, Joseph J, Shutinoski B, Startek $\mathrm{JB}$, et al. Type-I interferon signaling through ISGF3 complex is required for sustained Rip3 activation and necroptosis in macrophages. Proc Natl Acad Sci USA. 2014;111:E3206-E3213.

24. Huang Z, Wu S-Q, Liang Y, Zhou X, Chen W, Li L, et al. RIP1/ RIP3 binding to HSV-1 ICP6 initiates necroptosis to restrict virus propagation in mice. Cell Host Microbe. 2015;17:229-42.

25. Nogusa S, Thapa RJ, Dillon CP, Liedmann S, Oguin TH, Ingram JP, et al. RIPK3 activates parallel pathways of MLKL-driven necroptosis and FADD-mediated apoptosis to protect against influenza A virus. Cell Host Microbe. 2016;20:13-24.

26. Wang X, Li Y, Liu S, Yu X, Li L, Shi C, et al. Direct activation of RIP3/MLKL-dependent necrosis by herpes simplex virus 1 (HSV1) protein ICP6 triggers host antiviral defense. Proc Natl Acad Sci USA. 2014;111:15438-43.

27. Kitur K, Parker D, Nieto P, Ahn DS, Cohen TS, Chung S, et al. Toxin-induced necroptosis is a major mechanism of Staphylococcus aureus lung damage. PLoS Pathog. 2015;11:e1004820.

28. Pasparakis M, Vandenabeele P. Necroptosis and its role in inflammation. Nature. 2015;517:311-20.

29. Gunther C, Martini E, Wittkopf N, Amann K, Weigmann B, Neumann H, et al. Caspase-8 regulates TNF-alpha-induced epithelial necroptosis and terminal ileitis. Nature. 2011;477:335-9.
30. Kaiser WJ, Sridharan H, Huang C, Mandal P, Upton JW, Gough PJ, et al. Toll-like receptor 3-mediated necrosis via TRIF, RIP3, and MLKL. J Biol Chem. 2013;288:31268-79.

31. Otani T, Matsuda M, Mizokami A, Kitagawa N, Takeuchi H, Jimi E, et al. Osteocalcin triggers Fas/FasL-mediated necroptosis in adipocytes via activation of p300. Cell Death Dis. 2018;9:1194.

32. Jouan-Lanhouet S, Arshad MI, Piquet-Pellorce C, Martin-Chouly C, Le Moigne-Muller G, Van Herreweghe F, et al. TRAIL induces necroptosis involving RIPK1/RIPK3-dependent PARP-1 activation. Cell Death Differ. 2012;19:2003-14.

33. Wellington M, Koselny K, Sutterwala FS, Krysan DJ. Candida albicans triggers NLRP3-mediated pyroptosis in macrophages. Eukaryot Cell. 2014;13:329-40.

34. Uwamahoro N, Verma-Gaur J, Shen HH, Qu Y, Lewis R, Lu J, et al. The pathogen Candida albicans hijacks pyroptosis for escape from macrophages. MBio. 2014;5:e0003-00014.

35. Brown GD. Dectin-1: a signalling non-TLR pattern-recognition receptor. Nat Rev Immunol. 2006;6:33-43.

36. Dennehy KM, Brown GD. The role of the beta-glucan receptor Dectin-1 in control of fungal infection. $J$ Leukoc Biol. 2007;82:253-8.

37. Reid DM, Gow NA, Brown GD. Pattern recognition: recent insights from Dectin-1. Curr Opin Immunol. 2009;21:30-37.

38. Jouan-Lanhouet S, Riquet F, Duprez L, Vanden Berghe T, Takahashi N, Vandenabeele P. Necroptosis, in vivo detection in experimental disease models. Semin Cell Dev Biol. 2014;35:2-13.

39. Duprez L, Takahashi N, Van Hauwermeiren F, Vandendriessche B, Goossens V, Berghe TV, et al. RIP kinase-dependent necrosis drives lethal systemic inflammatory response syndrome. Immunity. 2011;35:908-18.

40. Kang T-B, Yang S-H, Toth B, Kovalenko A, Wallach D. Caspase8 blocks kinase RIPK3-mediated activation of the NLRP3 inflammasome. Immunity. 2013;38:27-40.

41. Wang X, Jiang W, Yan Y, Gong T, Han J, Tian Z, et al. RNA viruses promote activation of the NLRP3 inflammasome through a RIP1-RIP3-DRP1 signaling pathway. Nat Immunol. 2014;15:1126-33.

42. Lawlor KE, Khan N, Mildenhall A, Gerlic M, Croker BA, D’Cruz AA, et al. RIPK3 promotes cell death and NLRP3 inflammasome activation in the absence of MLKL. Nat Commun. 2015;6:6282.

43. Najjar M, Saleh D, Zelic M, Nogusa S, Shah S, Tai A, et al. RIPK1 and RIPK3 kinases promote cell-death-independent inflammation by Toll-like receptor 4. Immunity. 2016;45:46-59.

44. Newton K, Manning G. Necroptosis and Inflammation. Annu Rev Biochem. 2016;85:743-63.

45. Kaczmarek A, Vandenabeele P, Krysko DV. Necroptosis: the release of damage-associated molecular patterns and its physiological relevance. Immunity. 2013;38:209-23.

46. Daniels BP, Snyder AG, Olsen TM, Orozco S, Oguin TH, Tait SW, et al. RIPK3 restricts viral pathogenesis via cell deathindependent neuroinflammation. Cell. 2017;169:301-13. e311

47. Ofengeim D, Yuan J. Regulation of RIP1 kinase signalling at the crossroads of inflammation and cell death. Nat Rev Mol Cell Biol. 2013;14:727. 